\title{
A Scheduling Model of Isochronous Frame Generation on Self-similar Traffic of AOS Multiplexing
}

\author{
https://doi.org/10.3991/ijoe.v14i07.8203 \\ Yuntao Zhao, Lishi Liu( $\left.{ }^{\varpi}\right)$ \\ Shenyang Ligong University, Liaoning, China \\ liulishi2005@sina.com
}

\begin{abstract}
As the first layer of AOS multiplexing, the frame scheduling performance has a great effect on the efficiency of multiplexing. Traditional researches about the scheduling model mainly focus on the source packet arrival model of Poisson process. In this paper, an ON/OFF source arrival model with Pareto distribution of AOS multiplexing in self-similar service traffic is used to establish a isochronous frame-generated method through rigorous theoretical analysis, which results in a calculable formula of average packet delay and MPDU average multiplex efficiency. According to the simulation results, the simulation curve coincides with that of theory. The novel model contributes to the improvement of average packet delay and MPDU average multiplex efficiency on AOS.
\end{abstract}

Keywords-AOS, multiplexing, self-similar, Pareto distribution

\section{Introduction}

Consultative Committee for Space Data System (CCSDS) is an international standard organization, which composed of multinational space organization. And CCSDS established communication system structure, protocol and service for space data system. Advanced Orbit Systems (AOS) is a data transmit and communication mechanism, which also is a data processing and management system for space-to-space, space-to-surface measurement. AOS has changed the status of small capacity and low rate in space communication and construct a global, extensive, multidimensional data network [1-3].

AOS multiplexing technology includes frame generation technique and virtual channel dispatch technique [1-4], which allows the users to share one physical channel by time-division multiplexing, as is shown in Figure 1. As the first level of AOS multiplexing, the frame generation technique has a great effect on utilization efficiency of the multiplexing channel.

The frame generation in AOS, also known as packet channel multiplexing, is a mechanism of sharing virtual channels for all types of data generated from the upper layer [5]. It is to multiplex and encapsulate all kinds of data with same or similar demands in the service and to transmit packets in the same virtual channel into Multi- 
plexing Protocol Data Unit (MPDU) in a fixed length. The multiplexing model of AOS is shown in Fig 1.

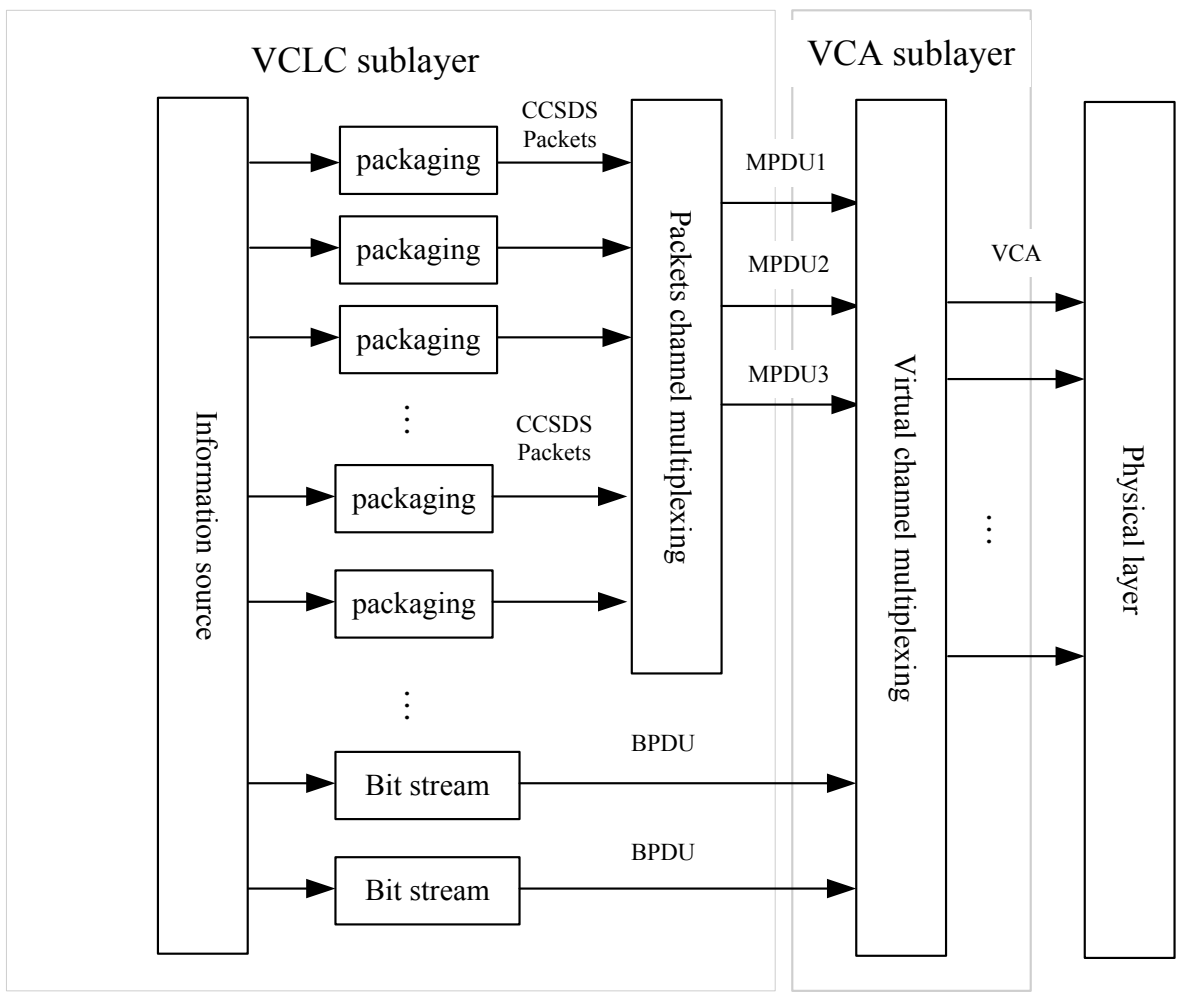

Fig. 1. Multiplexing Model of AOS

Recently, the researches on the frame generation of AOS have been paid more and more attention such as isochronous frame generation, high efficiency frame generation and self-similar frame generation. The isochronous frame generation [6] is to encapsulate the data packets from higher levels into a frame at a fixed interval time and then send them. It mainly focuses on the estimation [7], simulation [8], and analysis of MPDU multiplexing efficiency of AOS [9]. High efficiency frame generation means that the data packets will not be released until the overall length of the arrival data packets fill up the MPDU, whose multiplexing efficiency is 1 . And it focuses on the time of the frame generation and the analysis of the function of the packet time delay [10-11]. Ye Tian [11] puts forward an adaptive frame generation model. In this model a threshold value is set. With the value, as soon as the arrival packets fill up a frame, a frame is generated. If the arrival data packets are not enough to form a frame in a certain period of time, some empty data packets fill up the frame and are dispatched. In another paper [12], the author indicates that the adaptive model overcome long time delay problems, but which sometime cause the lower multiplexing efficien- 
cy than the isochronous frame generation. Since Leland [13] discovered the selfsimilarity of Ethernet service, many scientists have conducted a large number of researches one after another on the field and verified that various data packets exchanging network flow represents self-similarity. The self-similarity exists all the time no matter how the factors change, which are in topology structure, users, transmission medium, and protocol types etc. And the self-similarity has nothing to with when and where the service happens and the source encoding pattern [14]. The result of reference [15] shows that the flow of the terrestrial network generally presents selfsimilarity. Reference [16] further points out that not only the ground network traffic, but also the satellite network exhibits self-similarity. In the reference [17] it constructs a low earth orbit (LEO) satellite network model and confirms that the network also shows self-similarity on the simulation. The self-similar traffic model, as the source package arrival model, has been used to analysis the traffic of satellite network such as bandwidth allocation, service quality [18], assignment on demand multiple access [19-20], delay performance of the evaluation system [21]. The traffic of complex ground-space integration network also appears self-similarity, especially when it enters satellite links through earth-gate-stations [22-23]. Therefore, it is necessary to conduct the multiplexing research of AOS based on the self-similar traffic.

In the paper, a scheduling model of isochronous frame generation on self-similar traffic of AOS multiplexing is proposed. With ON/OFF source arrival model and Pareto distribution, a relational expression of average efficiency of MPDU multiplexing and a calculable formula of average packet delay are derived, which would contributes to the improvement of performance of AOS. According to the results, the simulation curve coincides with that of theory.

\section{The packet's arrival model on self-similar traffic}

\section{$2.1 \quad$ ON/OFF source}

Suppose that such a data source exists in a spatial data system, which sends data on the "ON" state and no data on the "OFF" state. The model is named as the "ON/OFF" model.

Let a series of "ON/OFF" sources $(1,2, \ldots M)$ be independent and the state space be $\{1,0,1, \mathrm{~L}\}$. As to any spatial state, each ON/OFF source is in any of the two states: the active ("ON") and the idle ("OFF"). In the active period, the transmission rate $R$ is stable; in the idle period, ON/OFF source doesn't transmit any data packet. The two states of "ON" and "OFF" change alternately. Such a data source is called the independent $\mathrm{ON} / \mathrm{OFF}$ source.

\subsection{The data packet arrival model}

The result of reference [24-25] shows the source packet arrival model produced by the superimposition of ON/OFF process can be described as the following: 
1. ON/OFF source arrival process is the Poisson flow with the parameter $\lambda$;

2. ON/OFF source arrival time span is subordinated to the Exponential distribution with the parameter $\lambda$;

3. ON/OFF source arrival period is subordinated to the Pareto distribution with the parameter $\alpha$.

The result of the ON/OFF source superimposition is a progressive self-similar traffic flow, and the corresponding Hurst coefficient is $H=(3-\alpha) / 2$.

The source packet arrival model produced by the superimposition of ON/OFF process is simulated as Figure 2.

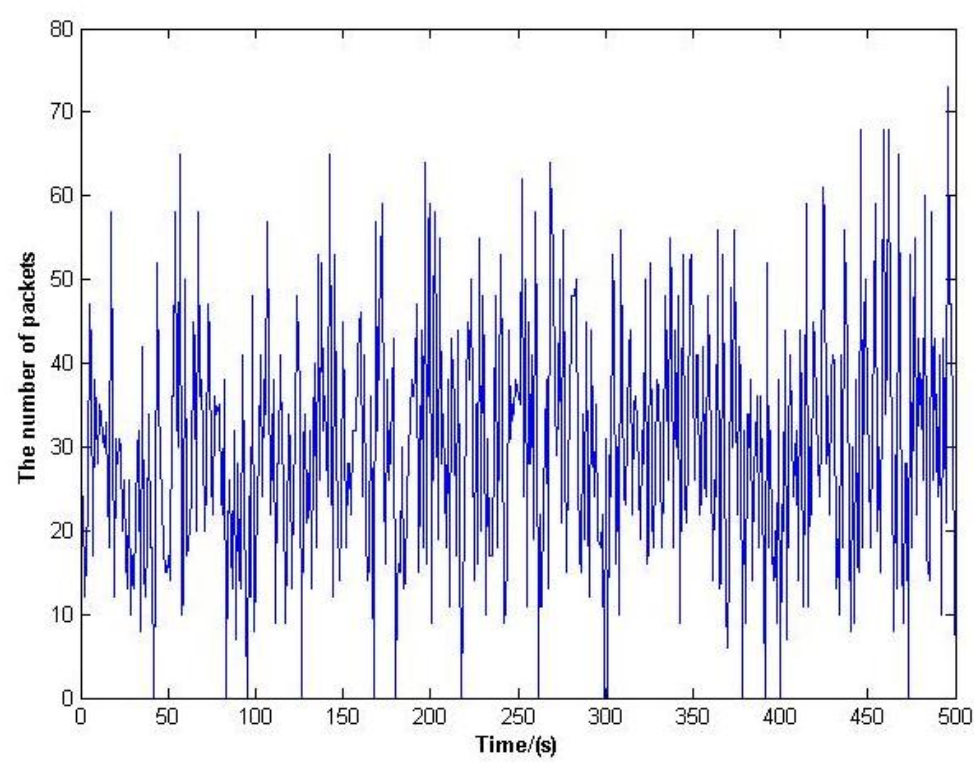

Fig. 2. Simulation of Packet Arrival Model Produced by Superimposition of ON/OFF Process

\section{The isochronous frame generation model in self-similar traffic}

\subsection{The description of the model}

The isochronous frame generation algorithm means that the data from the upper layer are encapsulated into a frame and sent in a fixed period. The fixed period is so called "isochronic".

In the paper, the discretization is adopted to study the MPDU multiplexing efficiency of the isochronous frame generation in self-similar traffic, which is discussed as the following. The notations in this paper are listed in Table 1. 
Table 1.

The notations table

\begin{tabular}{|c|c|}
\hline notation & The meanings expressed by a notation \\
\hline$\lambda$ & the parameter of the Poisson progress, the arrival rate \\
\hline$\alpha$ & the Pareto distribution parameter \\
\hline$H$ & Hurst coefficient \\
\hline$\xi_{t}$ & the arrival process of ON/OFF source In the period of $t$ \\
\hline$\tau_{t}$ & the duration of ON/OFF source in the period of $t$ \\
\hline$c$ & constant, decided by parameter $\alpha$ \\
\hline$\phi_{t}$ & the number of the arrival data packets in the period of $t$ \\
\hline$e f f_{m}$ & MPDU multiplexing efficiency \\
\hline$N$ & the length of MPDU \\
\hline$R$ & the transmission rate, constant \\
\hline$\phi$ & the number of the arrival data packets \\
\hline$P_{i, j}$ & the probability, $P_{i, j}=P\left(\xi_{t}=i, \tau_{t}=j\right)$ \\
\hline$\gamma_{i}$ & ON/OFF source arrival time interval \\
\hline$N_{t}$ & $\begin{array}{l}\text { the last count moment of ON/OFF source arrival interval or moment, } \\
\left\{N_{t}, \quad t \geq 0\right\} \text { is Poisson process }\end{array}$ \\
\hline$W_{k}$ & the ON/OFF source arrival moment \\
\hline$\xi$ & ON/OFF source arrival process \\
\hline$\phi_{1}$ & the sum of the packets transmitted in $\left(0, W_{1}\right)$ \\
\hline$r_{1 j}$ & the arrival moments of each packet in $\left(0, W_{1}\right)$ \\
\hline$\phi_{2}$ & the sum of the packets transmitted in, $\left(W_{1}, W_{2}\right)$ \\
\hline$r_{2 j}$ & the arrival moment of each packet in $\left(W_{1}, W_{2}\right)$ \\
\hline$a_{\tau}$ & the expectation of $\tau$ \\
\hline$T_{\sum 1}$ & the total packets time delay in $\left(0, W_{1}\right)$ \\
\hline$T_{\Sigma 2}$ & the total packets time delay in $\left(W_{1}, W_{2}\right)$ \\
\hline$T_{\Sigma}$ & the total packet time delay in $\left(0, N_{t}\right)$ \\
\hline$T_{\text {delay }}$ & The packet time delay of isochronous frame generation \\
\hline
\end{tabular}

In the period $t$, the arrival process $\xi_{t}$ of ON/OFF source is Poisson process, whose probability distribution is as the following. 


$$
P\left(\xi_{t}=k\right)=\frac{(\lambda t)^{k} \mathrm{e}^{-\lambda t}}{k !}, \quad k=0,1,2, \mathrm{~L}
$$

ON/OFF source's duration $\tau_{t}$ is subordinated to the Pareto distribution with parameter $\alpha$.

$$
P\left(\tau_{t}>t\right)=t^{-\alpha}, \quad i=1,2, \mathrm{~L}
$$

The discretization of formula (2) is as the following.

$$
P\left(\tau_{t}=j\right)=c j^{-\alpha-1}, \quad j=1,2 \mathrm{~L}
$$

$c$ is constant and its value is decided by parameter $\alpha$. The $j$ is the discretization value of time $\tau_{t}$. As $\xi_{t}$ and $\tau_{t}$ are independent, we have the following.

$$
P\left(\xi_{t}=i, \tau_{t}=j\right)=P\left(\xi_{t}=i\right) P\left(\tau_{t}=j\right)
$$

\subsection{The average efficiency of the isochronous frame generation}

In the period $t$, the number of the arrival data packets is marked as $\phi_{t}$, then $\phi_{t}=\xi_{t} \tau_{t} R$. MPDU multiplexing efficiency eff $f_{m}$ of AOS frame is defined as follows.

$$
\text { eff } f_{m}=\left\{\begin{array}{cl}
\frac{\xi_{t} \tau_{t} R}{N}, & \text { when } \xi_{t} \tau_{t} R<N \\
1, & \text { when } \xi_{t} \tau_{t} R \geq N
\end{array}\right.
$$

where $N$ is the length of MPDU, $R$ is the rate at which source generates data packets. It can be deduced by total probability formula.

$$
E\left(e f f_{m}\right)=\sum_{n=1}^{N-1} P\left(\xi_{t} \tau_{t} R=n\right) \frac{n}{N}+\sum_{n=N}^{\infty} P\left(\xi_{t} \tau_{t} R=n\right)
$$

The discrete results are listed, and the distribution of two-dimensional random variables $\left(\xi_{t}, \tau_{t}\right)$ is shown in table 2 .

Table 2. Probability of Two-dimensional Random Variables $\left(\xi_{t}, \tau_{t}\right)$

\begin{tabular}{|c|c|c|c|c|c|c|c|c|}
\hline$\left(\xi_{t}, \tau_{t}\right)$ & $(0,1)$ & $(0,2)$ & $\ldots$ & $(1,1)$ & $\ldots$ & $(2,1)$ & $(2,2)$ & $\ldots$ \\
\hline$\xi_{t} \tau_{t}$ & 0 & 0 & $\ldots$ & 1 & $\ldots$ & 2 & 4 & $\ldots$ \\
\hline $\mathrm{P}$ & $p_{0,1}$ & $p_{0,2}$ & $\ldots$ & $p_{1,1}$ & $\ldots$ & $p_{2,1}$ & $p_{2,2}$ & $\ldots$ \\
\hline
\end{tabular}

$P_{i, j}=P\left(\xi_{t}=i, \tau_{t}=j\right)$, and we have 


$$
\begin{gathered}
P\left(\xi_{t} \tau_{t}=0\right)=p_{0,1}+p_{0,2}+p_{0,3}+\mathrm{L}=c \mathrm{e}^{-\lambda t} \sum_{j=1}^{t} j^{-\alpha-1} \\
P\left(\xi_{t} \tau_{t}=k\right)=\sum^{m n=k} P_{m, n}, \quad k=1,2, \mathrm{~L}
\end{gathered}
$$

It can be gotten by the probability formula.

$$
E\left(e f f_{m}\right)=\sum_{n=1}^{N-1} P\left(\xi_{t} \tau_{t}=\frac{n}{R}\right) \frac{n}{N}+\sum_{n=N}^{\infty} P\left(\xi_{t} \tau_{t}=\frac{n}{R}\right)
$$

When $R \geq N \quad n=1,2, \ldots, N-1,0<\frac{n}{R}<1, P\left(\xi_{t} \tau_{t}=\frac{n}{R}\right)=0$. While $n=N, N+1, \ldots$. the value of $\frac{n}{R}$ is integer values 1,2,3,L . The formula (9) is arranged as the following.

$$
E\left(e f f_{m}\right)=\sum_{n=1}^{N-1} 0 \cdot \frac{n}{N}+\sum_{n=N}^{\infty} P\left(\xi_{t} \tau_{t} \geq 1\right)=1-P\left(\xi_{t} \tau_{t}=0\right)=1-c \mathrm{e}^{-\lambda t} \sum_{j=1}^{t} j^{-\alpha-1}
$$

When $R<N, n=0,1, \mathrm{~L} N-1$, we have

$$
P\left(\xi_{t} \tau_{t} R=n\right)=\sum_{n=1}^{N-1} P\left(\xi_{t} \tau_{t} \geq\left[\frac{N}{R}\right]\right)=\left\{\begin{array}{cc}
1-p_{00}-p_{11} & , 2 R>N>R \\
1-p_{00}-p_{11}-p_{12}-p_{21}, 3 R>N>2 R & \ldots
\end{array}\right.
$$

While $n=N, N+1, \ldots$. , the value of $\frac{n}{R}$ is integer such as $1,2,3, \mathrm{~L}$.

$$
\begin{aligned}
& E\left(e f f_{m}\right)=\sum_{n=1}^{N-1} P\left(\xi_{t} \tau_{t}=\frac{n}{R}\right) \frac{n}{N}+\sum_{n=N}^{\infty} P\left(\xi_{t} \tau_{t} \geq 1\right) \\
& =\sum_{n=1}^{N-1} P\left(\xi_{t} \tau_{t} \geq\left[\frac{N}{R}\right]\right) \frac{n}{N}+1-c \mathrm{e}^{-\lambda t} \sum_{j=1}^{t} j^{-\alpha-1}
\end{aligned}
$$

Generally, the value of $R$ will be bigger than $N$. The theoretical formula of average efficiency of the isochronous frame generation algorithm in self-similar traffic is gotten.

$$
E\left(e f f_{m}\right)=1-\mathrm{e}^{-\lambda t}\left(1-\frac{1}{\alpha t^{\alpha}}\right)=1-\mathrm{e}^{-\lambda t}+\frac{\mathrm{e}^{-\lambda t}}{\alpha t^{\alpha}}
$$

We use Matlab tools to simulate the above. The simulation parameters are set as the following. 
- the arrival rate $\lambda=0.2$ (package/second), exponential distribution parameter $\mu=1 / \lambda$; packet's length $l_{\mathrm{p}}=200$ bytes,

- the length of MPDU package area $l_{\mathrm{mp}}=2000 \mathrm{byte}$;

- the range of frame-forming period $S_{W}$ is $1 \sim 20 \mathrm{~s}$;

- the downstream rate of the self-similar traffic flow $R=30$ (package/second);

- Function of Pareto distribution $\operatorname{gprnd}(X, K, \sigma, \theta)$ with parameters $X=0.25, K=1.5, \sigma=\theta=1$. Here, $K$ corresponds to shape parameter $\alpha$, and $H=(3-\alpha) / 2$. Hurst coefficient of the self-similar traffic is 0.75 .

Figure 3 shows that under the condition of the isochronous frame generation algorithm in self-similar traffic. When the respective frame forming period $t$ is $10 \mathrm{~s}, 12 \mathrm{~s}$, $15 \mathrm{~s}, 20 \mathrm{~s}$, mean values of MPDU multiplexing efficiency are respectively 0.982 , $0.951,0.922,0.876$. Theoretically, the longer the waiting period is in frameforming, the higher MPDU multiplexing efficiency is. The simulation figure shows that MPDU multiplexing efficiency increases as time $t$ increases. The theoretical curve and the simulation curve coincide, which demonstrates the correctness of the reasoned theoretical formula.

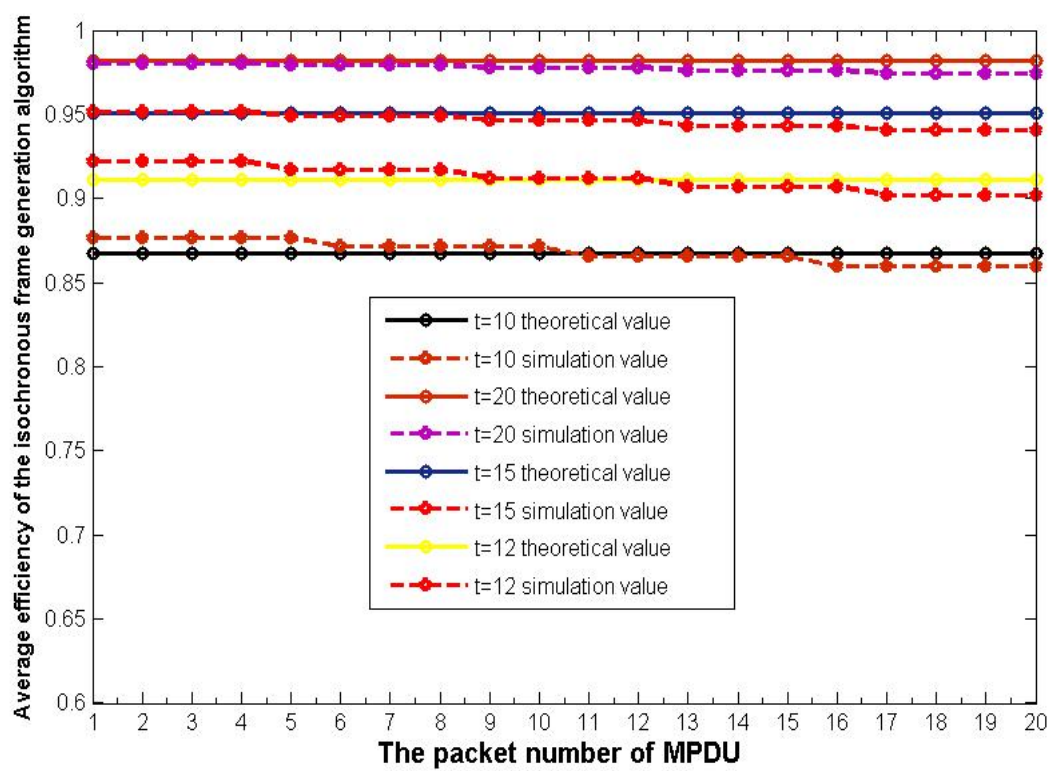

Fig. 3. The Average Value of MPDU Multiplexing Efficiency of the Isochronous Frame Generation Algorithm in Self-similar Traffic

In the traditional Poisson model, the researchers [9] analysis MPDU multiplexing efficiency of the isochronous frame generation by traditional Poisson model and gives the formula. But the formula is a recursion formula, which can't get the change of the efficiency with the parameter adjustment. The equation (13) in this paper is a closed- 
form solution, which shows that the MPDU multiplexing efficiency based on the traffic self-similar model is not only related to the parameters of the Poisson process, but also related to the parameters of the Pareto distribution. The simulation results show that the self-similar model can better reflect the actual traffic than traditional Poisson model. The comparison of two models is shown in the following Figure 4.

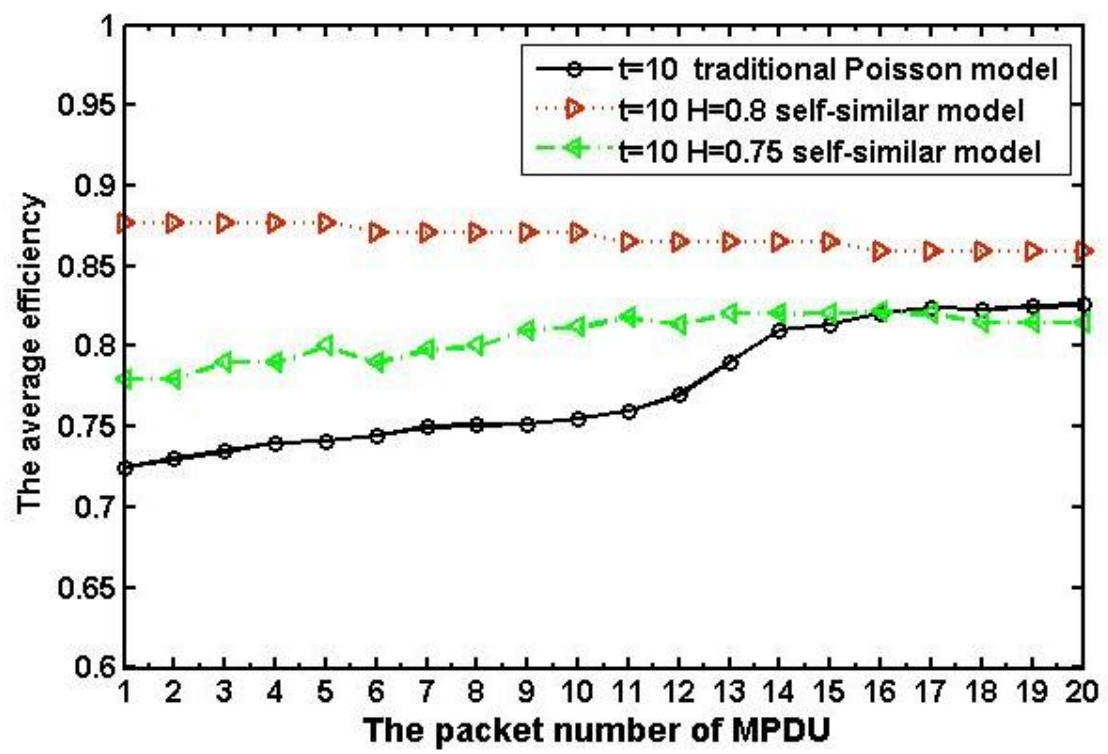

Fig. 4. The Comparison of the Traditional Poisson Model and the Self-similar Model in Terms of Efficiency

\subsection{The average time delay of the isochronous frame generation}

The previous researches based on the Poisson flow as the source packet arrival model mainly focused on MPDU multiplexing efficiency instead of the average packet time delay of the isochronous frame generation process. In the paper, the deduction has been conducted in the self-similar traffic.

The frame's generation period is $t$, and a random moment $t_{1}$ is chosen as the starting moment. Thus a frame is generated at the moment of $t_{1}+t$. The time delay of each packet includes two parts: one is the interval between two packets, and the other is time difference of the arrival time interval of ON/OFF source and the active period (In the period there is not an arrival packet). The former is a real time delay which exists in each frame, and the latter can be called virtual time delay. Because of the lack of the arrival packets (Packets are discarded even though they arrive, because MPDU has been full.), the arrival packets are still in the state of wait time. When the system is balanced ( $M \rightarrow \infty$ ), the virtual time delays can be distributed to each data packet. 
The system is correspondent to the following situations. During the arrival time $t$, let $\mathrm{ON} / \mathrm{OFF}$ source arrival time interval be $\gamma_{i}$, and $i=1,2, \ldots N_{t}$. Here $\left\{N_{t}, t \geq 0\right\}$ is Poisson process with the parameter $\lambda$ such as $E\left(\gamma_{i} \mid N_{t}=n\right)=\frac{t}{n+1}$. From the conclusion of the Poisson process there is

$$
E\left(N_{t}\right)=\lambda t
$$

Now suppose that in interval $(0, t)$ the ON/OFF source arrival moment is $W_{k}$, $k=1,2, \ldots N_{t}$. From the previous deduction $E\left(W_{k} \mid N_{t}=n\right)=\frac{k}{n+1} t, W_{k}$ obeys uniform distribution on $(0, t)$ under the condition of $N_{t}=n$.

Suppose that in $\left(0, W_{1}\right)$ the data packets are transmitted at the constant speed $R$ in the period $\tau_{1}$. The sum of the packets transmitted is $\phi_{1}=\xi_{1} \tau_{1} R$.

Let the arrival moments of each packet be $r_{11}, r_{12}, \ldots, r_{1 \phi_{1}}$ respectively.

$$
E\left(r_{1 j} \mid \phi_{1}=m\right)=E\left(\frac{j \tau}{m+1}\right)=\frac{j a_{\tau}}{m+1}, j=1,2, \ldots, m
$$

where $a_{\tau}$ is the expectation of $\tau_{i}$.

In $\left(0, W_{1}\right)$, let the expectation value of the total packets time delay be $E\left(T_{\Sigma^{1}}\right)$, and the deduction of $E\left(T_{\Sigma^{1}}\right)$ is as the following:

$$
\begin{aligned}
E\left(T_{\Sigma^{1}}\right) & =E\left(\sum_{k=1}^{\phi_{1}}\left(t-r_{1 k}\right)\right)=E\left(E\left(\sum_{k=1}^{\phi_{1}}\left(t-r_{1 k}\right) \mid \phi_{1}=n\right)\right) \\
& =\sum_{n=0}^{\infty} P\left(\phi_{1}=n\right) E\left(\left(n t-\sum_{k=1}^{\phi_{1}} r_{1 k}\right) \mid \phi_{1}=n\right) \\
& =\sum_{n=0}^{\infty} P\left(\phi_{1}=n\right)\left(n t-E\left(r_{11}+\ldots+r_{1 n}\right) \mid \phi_{1}=n\right) \\
& =\sum_{n=0}^{\infty} P\left(\phi_{1}=n\right)\left(n t-\frac{n a_{\tau}}{2}\right)=E\left(\phi_{1}\right)\left(t-\frac{a_{\tau}}{2}\right)
\end{aligned}
$$

In $\left(0, W_{1}\right)$ the average packet time delay is

$$
E\left(T_{Y_{1}}\right)=\frac{E\left(T_{\Sigma_{1}}\right)}{E\left(\phi_{1}\right)}=t-\frac{a_{\tau}}{2}
$$


In $\left(0, W_{2}\right)$ the sum of each time delay is calculated in $\left(W_{1}, W_{2}\right)$ with the same theory. Let $r_{21}, r_{22}, \ldots, r_{2 \phi_{2}}$ be the arrival moment of each packet in $\left(W_{1}, W_{2}\right)$.

$$
E\left(r_{2 j} \mid \phi_{2}=n\right)=E\left(W_{1}+\frac{j \tau_{2}}{n}\right)=E\left(W_{1}\right)+\frac{j a_{\tau}}{n+1}, j=1,2, \ldots, n
$$

It can be deduced as the following.

$$
\begin{gathered}
E\left(T_{\Sigma^{2}}\right)=E\left(\sum_{k=1}^{\phi_{2}}\left(t-r_{2 k}\right)\right)=E\left(E\left(\sum_{k=1}^{\phi_{2}}\left(t-r_{2 k}\right) \mid \phi_{2}=n\right)\right. \\
=\sum_{n=0}^{\infty} P\left(\phi_{2}=n\right) E\left(n t-\sum_{k=1}^{n} r_{2 k} \mid \phi_{2}=n\right) \\
=E\left(\phi_{2}\right)\left(t-E\left(W_{1}\right)-\frac{a_{\tau}}{2}\right)
\end{gathered}
$$

According to the same theory, we have

$$
\begin{gathered}
E\left(T_{Y_{2}}\right)=t-E\left(W_{1}\right)-\frac{a_{\tau}}{2} \\
E\left(T_{Y_{3}}\right)=t-E\left(W_{2}\right)-\frac{a_{\tau}}{2} \\
\cdots \cdots \\
E\left(T_{Y_{N_{t}}}\right)=t-E\left(W_{N_{t}-1}\right)-\frac{a_{\tau}}{2}
\end{gathered}
$$

With the formulas (17) and (22), the total average packet time delay in $\left(0, N_{t}\right)$ can be deduced as the following.

$$
\begin{aligned}
& E\left(T_{\Sigma}\right)=E\left(T_{Y_{1}}+T_{Y_{2}}+\ldots .+T_{Y_{Y_{N}}}\right)=E\left[\left(T_{Y_{1}}+T_{Y_{2}}+\ldots .+T_{Y_{N_{t}}}\right) \mid N_{t}\right] \\
& =\sum P\left(N_{t}=n\right) E\left[\left(T_{Y_{1}}+T_{Y_{2}}+\ldots .+T_{Y_{N_{t}}}\right) \mid N_{t}\right] \\
& =\sum P\left(N_{t}=n\right)\left(t-\frac{a_{\tau}}{2}+t-E\left(W_{1} \mid N_{t}=n\right)-\frac{a_{\tau}}{2}+\ldots+t-\frac{a_{\tau}}{2}-E\left(W_{n-1} \mid N_{t}=n\right)\right) \\
& =\sum P\left(N_{t}=n\right)\left(n t-n \frac{a_{\tau}}{2}-E \sum_{i=1}^{n-1}\left(W_{i} \mid N_{t}=n\right)\right) \\
& =\sum P\left(N_{t}=n\right)\left(n t-n \frac{a_{\tau}}{2}-(n-1) \frac{t}{2}\right)=E\left(N_{t}\right)\left(\frac{t}{2}-\frac{a_{\tau}}{2}\right)+\frac{t}{2}
\end{aligned}
$$


The average packet time delay of isochronous frame generation in self-similar traffic is deduced as the following.

$$
E\left(T_{\text {delay }}\right)=\frac{E\left(T_{\Sigma}\right)}{E\left(N_{t}\right)}=\frac{t}{2}-\frac{a_{\tau}}{2}+\frac{t}{2 \lambda t}=\frac{1}{2}\left(t-a_{\tau}+\frac{1}{\lambda}\right)
$$

When packet arrival rate $\lambda=0.2$ (packets/s), exponential distribution parameter $\mu=5$, the wait periods of frame-formation are $1.0 \mathrm{~s}, 1.2 \mathrm{~s}, 1.4 \mathrm{~s}, 1.6 \mathrm{~s}$ respectively. Theoretically the longer the wait time of frame is, the greater the average packet time delay of the isochronous frame generation. In Figure 4, the average packet time delay increases as the time $t$ grows. The theoretical curve and the simulation curve coincide, which demonstrates the availability of the deduced theoretical formula.

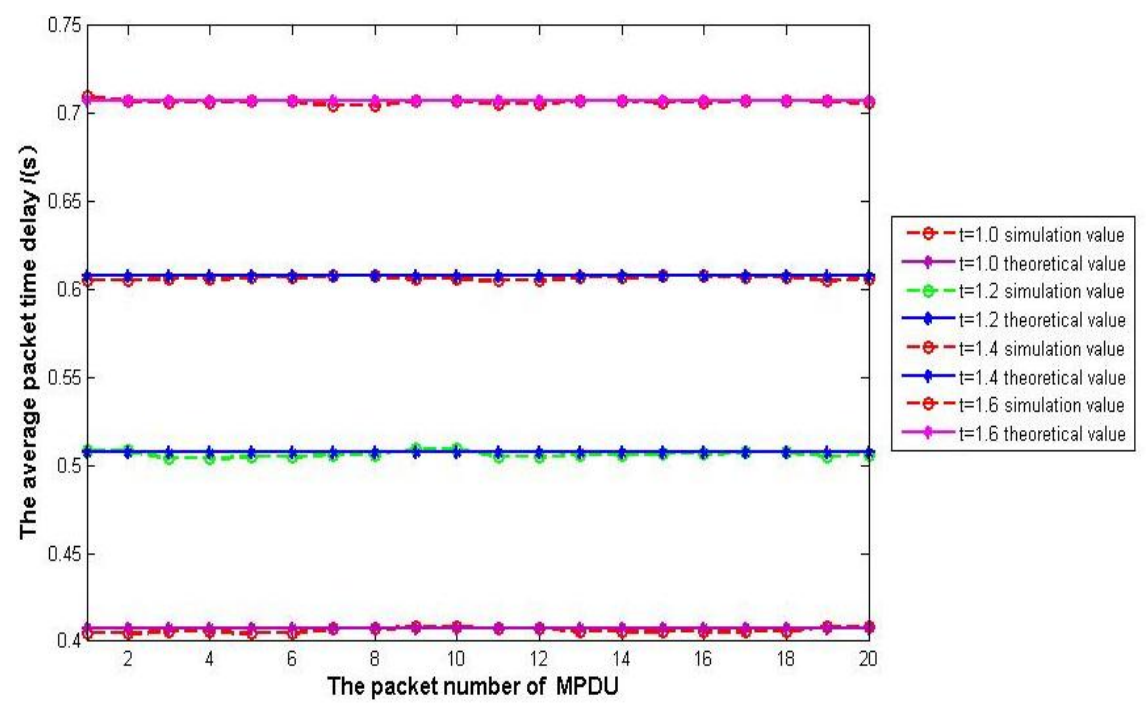

Fig. 5. The Average Delay of Isochronous Frame Generation in Self-similar Traffic

It is well known that, in traditional Poisson model, the average delay of isochronous frame generation is $t / 2$, that is nothing to do with $\lambda$. But in self-similar traffic model used in this paper, we can see from formula (24) that the average delay is not only related to $\lambda$ but also related to $a_{\tau}$. It shows that the self-similar model can better reflect the actual traffic of AOS than traditional Poisson model. The comparison of Poisson model and the self-similar model in terms of the average packet time delay is shown as Figure 6. 


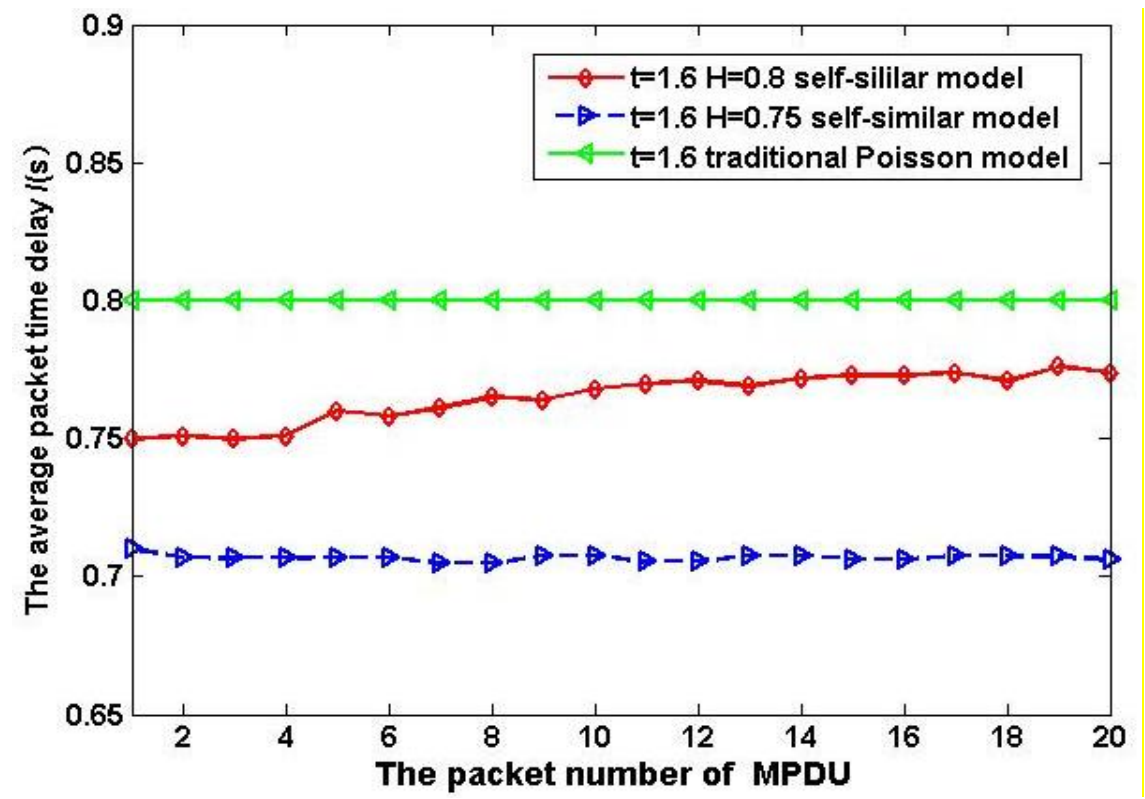

Fig. 6. The Comparison of Poisson Model and the Self-similar Model in Terms of the Average Packet Time Delay

\section{Conclusion}

The paper studies the function and mechanism of the multiplexing model of AOS protocols in different characteristic of self-similar traffic. In the paper a scheduling model of isochronous frame generation on self-similar traffic of AOS multiplexing is proposed. With a rigorous theoretical analysis, the average frame time delay with different arrival rates is studied. The calculating formula for the multiplexing efficiency of frame generation is gained, which would contributes to the improvement of performance of AOS. The experimental result shows that the simulation curve and the theoretical curve almost coincide, demonstrating the correctness of the deduced theoretical formula. And the calculating formulas show that the self-similar model can better reflect the actual situation than traditional Poisson model. Hence the research can provide theoretical support for the construction and operation of AOS development.

\section{$5 \quad$ Acknowledgement}

This work was supported by the National Natural Science Foundation of China (61471247, 61501307, 61101116, 61373159) , China Postdoctoral Science Foundation (2016M590234), Program for Liaoning Excellent Talents in University (LR2015057), General Project of Liaoning Provincial Department of Education 
(LG201611), Opening Foundation for Key Laboratory of Shenyang Ligong University(4771004kfs47, 4771004kfs32), and it also sponsored by "Liaoning BaiQianWan Talents Program(2014921044)", General Project of Liaoning Provincial Committee of Education (L2014078, L2015459), Project of Applied Basic Research of Shenyang(18-013-0-32).

\section{References}

[1] Liu Jianxun, Cheng Zijing, Lu Xiang, Lin Kai, Wang Chong. (2016). Research on Performance of Space and Ground Network Interconnection based on IP over AOS - Computer Engineering, 42:113-119.

[2] Long Yin, Zhu Ke, Chen Song, et al. (2016). A dynamic QoS assurance method for AOSJournal of Spacecraft TT\&C Technology, 35:161-168.

[3] Li Xiaoping. (2016). Study on Establishment of Operations Management System for Large-Scale Manufacturing Enterprise Based on AOS -Aeronautical Manufacturing Technology, 19:56-62.

[4] CCSDS. CCSDS 732.0-B-2.AOS space data link protocol. (2006). Washington. DC, USA: CCSDS Publishers.pp 1-87.

[5] Khan AA and Arsalan M. (2012). Implementation of CCSDS based imaging satellite packet processing unit on FPGA hardware. International Conference on Emerging Technologies IEEE (ICET). Pp1-6. https://doi.org/10.1109/ICET.2012.6375496

[6] Ba Yong. (2000). The analysis of CCSDS protocol and spatial data system. Doctoral Dissertation of Harbin School of Astronautics, Harbin Institute of Technology.pp 18-31.

[7] Bi MX, Pan CS and Zhao YT. (2009). Throughput analysis and frame length adaptive for AOS packet service. IEEE 2nd International Conference on Networks Security, Wireless Communication and Trusted Computing, Wuhan, China.pp 25-26.

[8] Zhang Yanqin. (2010). Research of the virtual channels scheduling algorithm based on advanced orbiting system. Dissertation of Shenyang Li gong University, Liaoning, China.

[9] Zhao Yuntao, Pan Chengsheng, Tian Ye, Zhang Zijing and Bi Mingxue. (2010). Research of the MPDU multiplexing efficiency based on CCSDS advanced orbiting system-Journal of Astronautics, 31: 1195-1199.

[10] Tian Ye, Zhang Deyu, Tan Zhenhui, Pan Chengsheng and Gao Xiaoling. (2011). Research on the packet time delay of frame generation algorithms in advanced orbiting systemsChinese High Technology Letters. 21: 1121-1128.

[11] Tian Ye, Pan Chengsheng, Zhang Zijing and Zhang Yanqin. (2011). Research on adaptive frame generation algorithm in AOS protocol-Journal of Astronautics, 32: 1171-1178.

[12] Tian Ye, Na Xin, Xia Ying, Gao Xiaoling and Liu Lishi. (2012). Research on the performance and application of adaptive frame generation algorithm in AOS protocol-Journal of Astronautics, 33: 242-248.

[13] Leland WE, Taqqut MS and Bellcore WW. (1994). On the self-similar nature of Ethernet traffic-Journal of IEEE/ACM Transaction on Networking, 2: 1-15.

[14] Crocell ME and Bestarros A. (1997). Self-similarity in World Wide Web traffic evidence and possible causes-Journal of IEEE/ACM Transactions on Networking, 5: 835-846.

[15] Ilow J and Leung H. (2001). Self-Similar texture modeling using FARIMA processes with applications to satellite images-Journal of. IEEE Transactions on Image Processing, 10: 792-797. https://doi.org/10.1109/83.918571

[16] Zhen Yuna, Gao Zihe and Guo Qing. (2007). Performance analysis of self-similar traffic in LEO satellite network. Proceedings of the sixth international conference on machine learning and cybernetics, Hong Kong, China, 5: 2649-2652. 
Paper-A Scheduling Algorithm of Isochronous Frame Generation on Self-similar Traffic of AOS Multi...

[17] Chiti F, Fantacci R and Tarchi D. (2005). QoS provisioning in GEO satellite with onboard processing using predictor algorithms-Journal of. IEEE Wireless Communications, 12: 2127. https://doi.org/10.1109/MWC.2005.1522100

[18] Wang Chengjin, Zhang Zhongquan, Han Yue. (2014). A New Bandwidth Estimation Algorithm for Self-similar Traffic Model-Computer Application and Software, 31:112-115.

[19] Shi Jingzhan. (2015). Research on MF_TDMA broadband satellite communication resource allocation algorithm based on business prediction-Dissertation of Nanjing University of Aeronautics and Astronautics.

[20] Wang Shi, Yang Huaijiang, Dong Yan.(2015). Prediction in LAN Traffic Flow Based on Chaos Theory-Journal of Beijing Institute of Technology, 36:616-619.

[21] SI Yuan, Chen Xin, Liu Zongqi.(2015). Bounding End to End Delay for Self-similar Traffic in LTE-A Femtocell Networks-Computer Science, 42:70-75.

[22] Na Zhenyu. (2010). Research on QoS Guarantee Methods in Satellite Internet-Dissertation of Harbin Institute of Technology.

[23] W Willinger, M. S. Taqqu, R. Sherman and D. V. Wilson. (1997). Self-similar through high-variability statistical analysis of Ethernet LAN traffic at the source level-Journal of ACM/Sigcomm .Computer Communication Review, 1:71-86.

[24] Norrs I. (1995). On the use of fractional Brownian motion in the theory of connectionless networks-IEEE Journal on Selected Areas in Communications, 13:953-962. https://doi.org/10.1109/49.400651

[25] Mao Yongcai and Hu Qiying. (2006). Stochastic process. Xidian University Publishers, China.pp 39-45.

[26] Hu Dihe. (2005). Stochastic process. Wuhan University Publishers, China.pp 141-143.

[27] Ranadheer Donthi, Ramesh Renikunta, Rajaiah Dasari and Malla Reddy Perati. Study of delay and loss behavior of internet Switch-Markovian modeling using Circulant Markov Modulated Poisson Process (CMMPP 2014), Journal of Applied Mathematics, vol. 5, issue 3, pp 512-519.

[28] Mutaz Beraka, Mohsin Iftikhar, Hassan Mathkour and Abdullah Bedaiwi. (2013). The exact analysis of limited service polling systems combined with non-preemptive priority scheduling under self-similar traffic input-Journal of Procedia Computer Science, vol. 19 , pp 214-223.

[29] Jin Shunfu, Yue Wuyi and Han Li. (2012). Performance analysis and evaluation of an enhanced power saving class type III in IEEE 802.16 with self-similar traffic-Journal of Global Optimization, 2:353-367. https://doi.org/10.1007/s10898-011-9681-6

\section{$7 \quad$ Authors}

Yuntao Zhao is an associate professor in Communication and Network Institute \& school of information science and Engineering, Shenyang Ligong University, Liaoning, 110159, China. His research interests include Space network protocol, Cyberspace Security (zhaoyuntao2014@163.com).

Lishi Liu is an associate professor in Communication and Network Institute \& school of science, Shenyang Ligong University, Liaoning, 110159, China. His research interests include Space link protocol (liulishi2005@sina.com).

Article submitted 06 January 2018. Resubmitted 13 January, 06 March, 27 March and 31 March 2018. Final acceptance 02 April 2018. Final version published as submitted by the authors. 